Research Article

\title{
Iterative Sliding Mode and Increment Feedback Attitude Control for On-Orbit Capturing Process of Spacecraft
}

\author{
ChunWu Yin, ${ }^{1,2}$ YouJun Ding $\mathbb{D}^{1}{ }^{1}$ LiJun Song, ${ }^{1}$ ZhaoQiang $\mathrm{Li}^{1}{ }^{1}$ and Xing $\mathrm{He}^{1}$ \\ ${ }^{1}$ School of Information and Control Engineering, Xi'an University of Architecture and Technology, Xi'an 710055, Shaanxi, China \\ ${ }^{2}$ ChuangLian Electronic Component (Group) Co., Ltd, Xi'an 710065, Shaanxi, China \\ Correspondence should be addressed to YouJun Ding; dyj@xauat.edu.cn
}

Received 21 November 2020; Revised 9 December 2020; Accepted 4 January 2021; Published 25 January 2021

Academic Editor: Gaoge $\mathrm{Hu}$

Copyright ( $\odot 2021$ ChunWu Yin et al. This is an open access article distributed under the Creative Commons Attribution License, which permits unrestricted use, distribution, and reproduction in any medium, provided the original work is properly cited.

According to the characteristics of spacecraft capturing noncooperative targets in orbit, an increment feedback controller based on nonlinear iterative sliding mode is presented. Firstly, the attitude tracking error equation is established, and then, an increment feedback control law based on bounded iterative sliding modes is proposed, which does not need to estimate the uncertain moment of inertia and external disturbances. For comparing, an adaptive sliding mode controller has been designed in the paper. Some numerical simulations have been given in the presence of spacecraft on-orbit capturing noncooperative target, and the simulation results show that the increment feedback controller has strong robustness to the unknown parametric variations and external disturbances and has a smaller control input torque in control process.

\section{Introduction}

With the increasing demand of communication and remote inspection, countless of satellites have been launched to space, and a large number of spacecraft debris have been generated simultaneously. In order to promote the sustainable development of the space and to ensure the safety of spacecraft on-orbit, the technology of spacecraft on-orbit acquisition has been proposed and studied by several researchers. On-orbit capturing technology has wide range of applications, such as cleaning space debris and repairing satellite, so the attitude stability controller of spacecraft capturing noncooperation target has an important practical application value. In the process of spacecraft on-orbit capturing noncooperation target, the moment of inertia of spacecraft will change due to the extension and recovery of manipulator. Thus, the essence of spacecraft stability control is attitude stability control under unknown inertial disturbance in the noncooperative process of spacecraft acquisition on-orbit.

The problem of spacecraft attitude control has been researched during the past decades, and most of control methods both linear and nonlinear have been provided.
The linear system linearizes the dynamics and kinematics equations of spacecraft at the equilibrium point, and the attitude stability controller for linear system is designed by classical linear control methods such as LQR control, H2 control, Ho control, and $\mathrm{H} 2 / \mathrm{H} \infty$ control based on representation form of quaternion; the spacecraft attitude motion equations are linearized by Silani and Lovera [1] and Yang [2,3]. Based on representation form of Euler angle, the spacecraft attitude motion equations are linearized by Yang and Sun [4]. The robustness and efficiency of all the classical linear control methods is compared through numerical simulations and practical applications by Silani and Lovera [1], Won [5], and Yang and Sun [4]. Nonlinear control such as PID-like control [6], backstepping control [7], sliding mode control [8-10], adaptive control [11, 12], inverse optimal control [13], fuzzy control [14], neural-network control [15], and other control methods [16] have been employed to design attitude stability controller, and Lyapunov theory has been utilized to analyze the stability of the whole system.

Some controllers only consider external disturbance, while others only compensate parametric disturbances. However, the focus of current research is on the design of 
attitude stabilization controllers with both parametric uncertainties and bounded external disturbances. Generally, Kalman filter [17-19], adaptive controller, or fuzzy/neuralnetwork approximator are used to estimate the disturbance of moment of inertia, and the sliding mode controller is applied to counteract external disturbance.

Considering the structure of adaptive controllers or sliding mode controllers, the numerical simulation results based on these controllers are analyzed, it is found that these controllers are complex, and the control input torque is large. The control input torque of the sliding mode adaptive controller designed by Yeh [10] is more than $2000 \mathrm{~N} \cdot \mathrm{m}$. The control torque of the controller designed by Silani and Lovera [1] is between $-50 \mathrm{~N} \cdot \mathrm{m}$ and $50 \mathrm{~N} \cdot \mathrm{m}$, and these control inputs do not satisfy the physical constraint of spacecraft.

Due to the physical and energy constraints, the actual system has state constraints and input saturation. Ignoring these restrictions, some undesired dynamic behaviors would occur in the practical control process. Therefore, the robust controller design with state and input constraint is still a challenge in the field of nonlinear system stability control $[20,21]$. In this paper, the design of robust attitude controller is discussed under the conditions of inertial matrix uncertainty, unknown disturbance, and control input constraints.

In this paper, an attitude stability controller based on iterative sliding mode and increment feedback is designed in the paper. In Section 2, the attitude tracking error equation is derived from attitude dynamics and kinematics equations of rigid spacecraft; in Section 3, the iterative sliding mode and increment feedback controller for attitude tracking error system are proposed. Based on Lyapunov stability theory, the closed-loop system is proved to be exponentially convergent. In Section 4, the adaptive sliding mode attitude stability controller is designed for comparison. In Section 5, the control performance is verified by numerical simulations. Finally, the proposed method is demonstrated by numerical simulation in Section 6.

\section{Attitude Dynamics of a Rigid Spacecraft}

2.1. Dynamics Equation. The attitude dynamic motion of rigid spacecraft with the external disturbance can be described by the following set of differential equation:

$$
\mathbf{J} \dot{\omega}=-\boldsymbol{\omega}^{\times} \mathrm{J} \boldsymbol{\omega}+\tau_{u}+\tau_{d},
$$

where $\mathbf{J} \in \mathbf{R}^{3 \times 3}$ denotes the inertia matrix of the body and satisfies $\mathbf{J}=\mathbf{J}^{T}>0, \omega \in \mathbf{R}^{3}$ is the angular velocity vector of the body in the body-fixed frame, $\tau_{u} \in \mathbf{R}^{3}$ is the control torque vector, and $\tau_{d} \in \mathbf{R}^{3}$ is the bounded external disturbance vector such as gravity and radiation. Moreover, the matrix $\omega^{\times} \in \mathbf{R}^{3 \times 3}$ denotes a skew symmetric matrix given by

$$
\boldsymbol{\omega}^{\times}=\left[\begin{array}{ccc}
0 & \omega_{3}(t) & -\omega_{2}(t) \\
-\omega_{3}(t) & 0 & \omega_{1}(t) \\
\omega_{2}(t) & -\omega_{1}(t) & 0
\end{array}\right] .
$$

2.2. Kinematics Equation. The orientation of rigid spacecraft can be described by several parameterizations with a special orthogonal group. In this paper, the orientation of the rigid body is parameterized by the yaw-pitch-roll $\left(\theta_{3}-\theta_{2}-\theta_{1}\right)$ Euler angle. The attitude kinematics equation of rigid spacecraft is described as follows:

$$
\boldsymbol{\omega}=R(\boldsymbol{\theta}) \dot{\theta}-\boldsymbol{\omega}_{c}(\boldsymbol{\theta}),
$$

where $\theta=\left[\theta_{1}, \theta_{2}, \theta_{3}\right]^{\mathrm{T}}$ is the attitude Euler angles and $\omega_{0}$ is the orbital rate. Also,

$$
\begin{aligned}
\mathbf{R}(\boldsymbol{\theta}) & =\left(\begin{array}{ccc}
1 & 0 & -\sin \theta_{2} \\
0 & \cos \theta_{1} & \sin \theta_{1} \cos \theta_{2} \\
0 & -\sin \theta_{1} & \cos \theta_{1} \cos \theta_{2}
\end{array}\right), \\
\boldsymbol{\omega}_{c}(\boldsymbol{\theta}) & =\omega_{0}\left[\begin{array}{c}
\cos \theta_{2} \sin \theta_{3} \\
\cos \theta_{1} \sin \theta_{3}+\sin \theta_{1} \sin \theta_{2} \sin \theta_{3} \\
-\sin \theta_{1} \cos \theta_{3}+\cos \theta_{1} \sin \theta_{2} \sin \theta_{3}
\end{array}\right] .
\end{aligned}
$$

Taking the time derivative of (3), the following is obtained:

$$
\dot{\omega}=\mathbf{R}(\boldsymbol{\theta}) \ddot{\theta}+\frac{d}{\mathrm{~d} t} \mathbf{R}(\boldsymbol{\theta}) \dot{\theta}-\frac{d}{\mathrm{~d} t} \boldsymbol{\omega}_{\mathrm{c}}(\boldsymbol{\theta}) .
$$

Substituting (3) and (5) into (1) and premultiplying (5) by the matrix $\mathbf{R}^{\mathbf{T}}(\theta)$, the following is obtained:

$$
\mathbf{M}(\boldsymbol{\theta}) \ddot{\theta}+\mathbf{C}(\boldsymbol{\theta}, \dot{\theta}) \dot{\theta}+\mathbf{G}(\boldsymbol{\theta}, \dot{\theta})=\mathbf{u}+\mathbf{d},
$$

where

$$
\begin{aligned}
\mathbf{M}(\boldsymbol{\theta}) & =\mathbf{R}^{\mathrm{T}}(\boldsymbol{\theta}) \mathbf{J} \mathbf{R}(\boldsymbol{\theta}) \\
\mathbf{C}(\boldsymbol{\theta}, \dot{\theta}) & =\mathbf{R}^{\mathrm{T}}(\boldsymbol{\theta}) \mathbf{J} \frac{\mathbf{d}}{\mathbf{d t}} \mathbf{R}(\boldsymbol{\theta})+\mathbf{R}^{\mathrm{T}}(\boldsymbol{\theta}) \boldsymbol{\omega}^{\times} \mathbf{J R}(\boldsymbol{\theta}), \\
\mathbf{G}(\boldsymbol{\theta}, \dot{\theta}) & =-\mathbf{R}^{\mathrm{T}}(\boldsymbol{\theta}) \mathbf{J} \frac{\mathbf{d}}{\mathbf{d t}} \boldsymbol{\omega}_{\mathbf{c}}(\boldsymbol{\theta})-\mathbf{R}^{\mathrm{T}}(\boldsymbol{\theta}) \boldsymbol{\omega}^{\times} \mathbf{J} \boldsymbol{\omega}_{\mathbf{c}}(\boldsymbol{\theta}), \\
\mathbf{u} & =\mathbf{R}^{\mathrm{T}}(\boldsymbol{\theta}) \boldsymbol{\tau}_{\mathbf{a}} \\
\mathbf{d} & =\mathbf{R}^{\mathrm{T}}(\boldsymbol{\theta}) \boldsymbol{\tau}_{\mathbf{d}}
\end{aligned}
$$

Denote $\mathbf{J}_{0}$ as nominal moment of inertia and $\Delta \mathbf{J}$ as inertia uncertainties, then the real moment of inertia is $\mathbf{J}=\mathbf{J}_{0}+\Delta \mathbf{J}$.

Accordingly, there are

$$
\begin{aligned}
\mathbf{M}(\boldsymbol{\theta}) & =\mathbf{R}^{\mathrm{T}}(\boldsymbol{\theta}) \mathbf{J}_{0} \mathbf{R}(\boldsymbol{\theta})+\mathbf{R}^{\mathrm{T}}(\boldsymbol{\theta}) \Delta \mathbf{J} \mathbf{R}(\boldsymbol{\theta}) \\
& =\mathbf{M}_{0}(\boldsymbol{\theta})+\Delta \mathbf{M}(\boldsymbol{\theta}), \\
\mathbf{C}(\boldsymbol{\theta}, \dot{\theta}) & =\mathbf{C}_{0}(\boldsymbol{\theta}, \dot{\theta})+\Delta \mathbf{C}(\boldsymbol{\theta}, \dot{\theta}) .
\end{aligned}
$$

Substituting (8) and (9) into (6) yields

$$
\begin{aligned}
\mathbf{M}_{0}(\boldsymbol{\theta}) \ddot{\theta} & +\mathbf{C}_{0}(\boldsymbol{\theta}, \dot{\theta}) \dot{\theta}=\mathbf{u}+\mathbf{d}-\Delta \mathbf{M}(\boldsymbol{\theta}) \ddot{\theta} \\
& -\Delta \mathbf{C}(\boldsymbol{\theta}, \dot{\theta}) \dot{\theta}-\mathbf{G}(\boldsymbol{\theta}, \dot{\theta}) .
\end{aligned}
$$




$$
\mathbf{Y}=-\Delta \mathbf{M}(\boldsymbol{\theta}) \ddot{\theta}-\Delta \mathbf{C}(\boldsymbol{\theta}, \dot{\theta}) \dot{\theta}-\mathbf{G}(\boldsymbol{\theta}, \dot{\theta})
$$

Then, equation (10) becomes

$$
\ddot{\theta}=\mathbf{M}_{0}^{-1}(\boldsymbol{\theta})\left(\mathbf{u}+\mathbf{d}+\mathbf{Y}-\mathbf{C}_{0}(\boldsymbol{\theta}, \dot{\theta}) \dot{\theta}\right) .
$$

2.3. Attitude Tracking Error Equation. Denote the desired attitude angle as $\theta_{r} \in C^{2}$; correspondingly, its desired velocity vector is $\dot{\theta}_{r}$ and acceleration vector is $\ddot{\theta}_{r}$. Denote attitude tracking error as

$$
\mathbf{e}_{1}=\boldsymbol{\theta}-\boldsymbol{\theta}_{r}
$$
(12):

Let $\mathbf{e}_{2}=\dot{e}_{1}=\dot{\theta}-\dot{\theta}_{r}$, taking the time derivative of $\mathbf{e}_{2}$ with

$$
\begin{aligned}
\dot{e}_{2} & =\ddot{\theta}-\ddot{\theta}_{r}=\mathbf{M}_{0}^{-1}\left(\mathbf{u}+\mathbf{d}+\mathbf{Y}-\mathbf{C}_{0} \dot{\theta}\right)-\ddot{\theta}_{r} \\
& =\mathbf{M}_{0}^{-1}\left(\mathbf{u}+\mathbf{d}+\mathbf{Y}-\mathbf{C}_{0} \mathbf{e}_{2}-\mathbf{C}_{0} \dot{\theta}_{r}\right)-\ddot{\theta}_{r} .
\end{aligned}
$$
as

Then, it will obtain the attitude tracking error equations

$$
\left\{\begin{array}{l}
\dot{e}_{1}=\mathbf{e}_{2}, \\
\dot{e}_{2}=-\mathbf{M}_{0}^{-1} \mathbf{C}_{0} \mathbf{e}_{2}-\mathbf{M}_{0}^{-1} \mathbf{C}_{0} \dot{\theta}_{r}-\ddot{\theta}_{r}+\mathbf{M}_{0}^{-1}(\mathbf{u}+\mathbf{d}+\mathbf{Y}) .
\end{array}\right.
$$

The attitude control objective of spacecraft on-orbit capturing noncooperative target is to design the control law of system (15) to ensure global asymptotic stability of the closed-loop system in the presence of uncertain moment inertia matrix $\Delta \mathbf{J}$, unknown disturbance $\mathbf{d}$, and state and input constraints.

\section{Adaptive Sliding Mode Attitude Stable Controller Design}

3.1. Controller Design. When the parameter perturbation satisfies the matching conditions, the sliding mode control has good robustness due to the invariance of the system. Increment feedback control has simple structure and small control torque because the parameter perturbance and external disturbance are directly contained into the controller. Therefore, a nonlinear iterative sliding mode attitude stability control law is designed based on increment feedback control in this section. Design PID attitude controller (16) for system (15):

$$
\mathbf{u}=-k_{d} \dot{e}_{1}-k_{p} \mathbf{e}_{1}-k_{i} \int \mathbf{e}_{1} \mathrm{~d} t
$$

where const $k_{p} \in R^{+}, k_{d} \in R^{+}$, and $k_{i} \in R^{+}$.

Modify the controller (16)

$$
\begin{aligned}
\mathbf{u} & =-k_{d} \dot{e}_{1}-k_{p} \mathbf{e}_{1}-k_{i} \int \mathbf{e}_{1} \mathrm{~d} t \\
& =-k_{d}\left[\dot{\mathrm{e}}_{1}+\left(\frac{k_{p}}{2 k_{d}}+\sqrt{\frac{k_{p}^{2}}{4 k_{d}^{2}}-\frac{k_{i}}{k_{d}}}\right) \mathrm{e}_{1}+\left(\frac{k_{p}}{2 k_{d}}-\sqrt{\frac{k_{p}^{2}}{4 k_{d}^{2}}-\frac{k_{i}}{k_{d}}}\right) \mathbf{e}_{1}+\left(\frac{k_{p}}{2 k_{d}}+\sqrt{\frac{k_{p}^{2}}{4 k_{d}^{2}}-\frac{k_{i}}{k_{d}}}\right)\left(\frac{k_{p}}{2 k_{d}}-\sqrt{\frac{k_{p}^{2}}{4 k_{d}^{2}}-\frac{k_{i}}{k_{d}}}\right) \int \mathbf{e}_{1} \mathrm{~d} t\right]
\end{aligned}
$$

Let $\quad k_{1}=\left(\left(k_{p} / 2 k_{d}\right)-\sqrt{\left(k_{p}^{2} / 4 k_{d}^{2}\right)-\left(k_{i} / k_{d}\right)}\right), k_{2}=\left(\left(k_{p} /\right.\right.$ $\left.\left.2 k_{d}\right)+\sqrt{\left(k_{p}^{2} / 4 k_{d}^{2}\right)-\left(k_{i} / k_{d}\right)}\right)$ and obtain

$$
\begin{aligned}
\mathbf{u} & =-k_{d}\left[\dot{e}_{1}+k_{1} \mathbf{e}_{1}+k_{2} \mathbf{e}_{1}+k_{1} k_{2} \int \mathbf{e}_{1} \mathrm{~d} t\right] \\
& =-k_{d}\left[\dot{e}_{1}+k_{1} \mathbf{e}_{1}+k_{2}\left(\mathbf{e}_{1}+k_{1} \int \mathbf{e}_{11} \mathrm{~d} t\right)\right] \\
& =-k_{d}\left[\frac{d}{\mathrm{~d} t}\left(\mathbf{e}_{1}+k_{1} \int \mathbf{e}_{1} \mathrm{~d} t\right)+k_{2}\left(\mathbf{e}_{1}+k_{1} \int \mathbf{e}_{1} \mathrm{~d} t\right)\right] .
\end{aligned}
$$

Define $\mathbf{e}_{0}=\int \mathbf{e}_{1} \mathrm{~d} t$, then $\dot{e}_{0}=\mathbf{e}_{1}$; equation (15) can be modified as

$$
\left\{\begin{array}{l}
\dot{e}_{0}=\mathbf{e}_{1}, \\
\dot{e}_{1}=\mathbf{e}_{2}, \\
\dot{e}_{2}=-\mathbf{M}_{0}^{-1} \mathbf{C}_{0} \mathbf{e}_{2}-\mathbf{M}_{0}^{-1} \mathbf{C}_{0} \dot{\theta}_{r}-\ddot{\theta}_{r}+\mathbf{M}_{0}^{-1}(\mathbf{u}+\mathbf{d}+\mathbf{Y}) .
\end{array}\right.
$$

Substituting $\mathbf{e}_{0}=\int \mathbf{e}_{1} \mathrm{~d} t$ in equation (18), the following is obtained:

$$
\mathbf{u}=-k_{d}\left[\frac{d}{\mathrm{~d} t}\left(e_{1}+k_{1} \mathbf{e}_{0}\right)+k_{2}\left(\mathbf{e}_{1}+k_{1} \mathbf{e}_{0}\right)\right] .
$$

Define variates as follows:

$$
\left\{\begin{array}{l}
\boldsymbol{\sigma}_{1}=k_{1} \mathbf{e}_{0}+\dot{e}_{0} \\
\boldsymbol{\sigma}_{2}=k_{2} \boldsymbol{\sigma}_{1}+\dot{\sigma}_{1}
\end{array}\right.
$$

where $\sigma_{1}$ is a sliding mode on the phase plane $\mathbf{e}_{0} \dot{e}_{0}, \dot{e}_{0}=$ $-k_{1} \mathbf{e}_{0}$ as $\sigma_{1} \longrightarrow 0, \mathbf{e}_{0}$ will converge exponentially, and $\sigma_{2}$ is a sliding mode on the phase plane $\sigma_{1} \dot{\sigma}_{1}$. When $\sigma_{2} \longrightarrow 0$, it can obtain $\dot{\sigma}_{1}=-k_{2} \sigma_{1}$, and $\sigma_{2}$ also will converge exponentially, so the stability of system (15) is equal to the stabilization control of $\sigma_{2}$.

From the above analysis, the iterative design method of spacecraft attitude control can be obtained. Firstly, the sliding mode $\sigma_{1}$ based on tracking errors $\mathbf{e}_{1}$ and $\dot{e}_{1}$ can be designed for system (15) to obtain a new system. Then, 
another sliding mode $\sigma_{2}$ can be designed for the new system based on $\sigma_{1}$ and $\dot{\sigma}_{1}$.

In order to satisfy the constraints of bounded state and control input and to eliminate the uncertainties caused by parameter perturbations and external disturbances, the nonlinear interactive sliding mode is designed as follows:

$$
\left\{\begin{array}{l}
\boldsymbol{\sigma}_{1}=k_{1} \tanh \left(k_{2} \mathbf{e}_{1}\right)+\dot{e}_{1}, \\
\boldsymbol{\sigma}_{2}=k_{3} \tanh \left(k_{4} \boldsymbol{\sigma}_{1}\right)+\dot{\sigma}_{1},
\end{array}\right.
$$

where const $k_{1}, k_{2}, k_{3}, k_{4}>0 . \tanh (\cdot)$ is the hyperbolic tangent function, and it can limit the range of the variable. $\sigma_{1}$ is a sliding mode on the phase plane $\mathbf{e}_{1} \dot{e}_{1}, \dot{e}_{1}=-k_{1} \tanh \left(k_{2} \mathbf{e}_{1}\right)$ as $\sigma_{1} \longrightarrow 0$; when the deviation of $\mathbf{e}_{1}$ is big, $\mathbf{e}_{1}$ will convergence with a fixed rate(i.e., $k_{1}$ ), and when the deviation of $\mathbf{e}_{1}$ is small, $\mathbf{e}_{1}$ will converge exponentially; $k_{2}$ is a compression or tension coefficient, which determines the sliding slope in the origin of phase plane together with $k_{1} . \sigma_{2}$ is a sliding mode on the phase plane $\sigma_{1} \dot{\sigma}_{1}$; when $\sigma_{2} \longrightarrow 0$, it can obtain $\dot{\sigma}_{1}=-k_{3} \tanh \left(k_{4} \sigma_{1}\right)$; when the deviation of $\sigma_{1}$ is big, $\sigma_{1}$ will converge with a fixed rate approximately(i.e., $k_{3}$ ); if the deviation of $\sigma_{1}$ is small, $\sigma_{1}$ will converge exponentially; from the definition of $\sigma_{1}$ and $\sigma_{2}$, we can obtain that the stability control of system (15) is equal to the stability control of $\sigma_{2}$.

Taking the time derivative of $\sigma_{1}$, give

$$
\begin{aligned}
\dot{\sigma}_{1}= & k_{1}\left[\frac{4}{\left(e^{k_{2} e_{1}}+e^{-k_{2} e_{1}}\right)^{2}}\right]^{\mathrm{T}} k_{2} \dot{e}_{1}+e_{1} \\
= & k_{1}\left[\frac{4}{\left(e^{k_{2} e_{1}}+e^{-k_{2} e_{1}}\right)^{2}}\right]^{\mathrm{T}} k_{2} \dot{e}_{1}-\mathbf{M}_{0}^{-1} \mathbf{C}_{0} \mathbf{e}_{2}- \\
& \mathbf{M}_{0}^{-1} \mathbf{C}_{0} \dot{\theta}_{r}-\theta_{r}+\mathbf{M}_{0}^{-1}(\mathbf{u}+\mathbf{d}+\mathbf{Y}) .
\end{aligned}
$$

Substituting (23) into (22) yields

$$
\begin{aligned}
\boldsymbol{\sigma}_{2}= & k_{3} \tanh \left(k_{4} k_{1} \tanh \left(k_{2} \mathbf{e}_{1}\right)+k_{4} \mathbf{e}_{2}\right) \\
& +k_{1}\left[\frac{4}{\left(e^{k_{2} e_{1}}+e^{-k_{2} e_{1}}\right)^{2}}\right]^{\mathrm{T}} k_{2} \dot{e}_{1} \\
& -\mathbf{M}_{0}^{-1} \mathbf{C}_{0} \mathbf{e}_{2}-\mathbf{M}_{0}^{-1} \mathbf{C}_{0} \dot{\theta}_{r}-\ddot{\theta}_{r}+\mathbf{M}_{0}^{-1}(\mathbf{u}+\mathbf{d}+\mathbf{Y}) .
\end{aligned}
$$

In order to avoid the estimation of the unknown moment of inertia and unknown external disturbance and simplify the implementation of attitude stability controller, the following incremental feedback controller is designed to ensure the stability of zero-order scalar system (24):

$$
\begin{aligned}
\dot{u} & =-k_{p} \sigma_{2}-\varepsilon s a t\left(\sigma_{2}\right), \\
\operatorname{sat}(x) & = \begin{cases}x_{\text {min }}, & \text { if } x<x_{\min }, \\
x, & \text { if } x_{\min } \leq x \leq x_{\max }, \\
x_{\max }, & \text { if } x>x_{\max },\end{cases}
\end{aligned}
$$

where const $k_{p}, \varepsilon \in R^{+} . x_{\min }$ and $x_{\max }$ are the minimum value and maximum value of $x$, respectively. The incremental feedback controller does not need to estimate or cancel unknown moment of inertia and unknown external disturbance but only needs to search the solution of $\sigma_{2}=0$ along with the opposite direction of $\sigma_{2}$, which avoids the waste of control input energy and reduces the magnitude of the control input torque effectively.

3.2. Analysis of the Stability. Barbalat Lemma: if a function $f(t)$ is continuous and differentiable and satisfies the following:

(1) $\lim _{t \rightarrow \infty} f(t)=C$ C is bounded constant.

(2) $\dot{f}(t)$ is uniformly continuous (means $\ddot{f}(t)$ is bounded), then $\lim _{t \rightarrow \infty} \dot{f}(t)=0$.

Theorem 1. Under the iterative sliding mode (22) and incremental feedback controller (25), the closed-loop system (15) is globally asymptotic stable.

Proof. According to the above analysis, it is known that the stability control of system (15) is equal to the stability control of $\sigma_{2}$.

Considering Lyapunov function as $V=(1 / 2) \sigma_{2}^{\mathrm{T}} \sigma_{2}$ and taking the time derivative of $V$ yields

$$
\dot{V}=\boldsymbol{\sigma}_{2}^{\mathrm{T}} \dot{\sigma}_{2}=\boldsymbol{\sigma}_{2}^{\mathrm{T}} \frac{\partial \boldsymbol{\sigma}_{2}}{\partial \mathbf{u}} \dot{u} .
$$

Ignoring the variables of independent of $\mathbf{u}$ in (24) and taking the time derivative of (24) about $\mathbf{u}$, we obtain

$$
\frac{\partial \boldsymbol{\sigma}_{2}}{\partial \mathbf{u}}=\mathbf{M}_{0}^{-1}
$$

Because $\mathbf{M}_{0}$ is a positive matrix, $\mathbf{M}_{0}^{-1}$ is also a positive matrix. Substituting (25) and (27) into (26) yields

$$
\begin{aligned}
\dot{V} & =\boldsymbol{\sigma}_{2}^{\mathrm{T}} \mathbf{M}_{0}^{-1}\left[-k_{p} \boldsymbol{\sigma}_{2}-\varepsilon \mathbf{s a t}\left(\boldsymbol{\sigma}_{2}\right)\right] \\
& =-k_{p} \boldsymbol{\sigma}_{2}^{\mathrm{T}} \mathbf{M}_{0}^{-1} \boldsymbol{\sigma}_{2}-\varepsilon \boldsymbol{\sigma}_{2}^{\mathrm{T}} \mathbf{M}_{0}^{-1} \operatorname{sat}\left(\boldsymbol{\sigma}_{2}\right) \\
& \leq-k_{p} \boldsymbol{\sigma}_{2}^{\mathrm{T}} \mathbf{M}_{0}^{-1} \boldsymbol{\sigma}_{2} \leq 0 .
\end{aligned}
$$

Then, we can obtain $\dot{V}=\sigma_{2}^{\mathrm{T}} \dot{\sigma}_{2} \leq 0$, which means $\dot{V} \leq 0$ when $t \geq 0$. According to the Barbalat lemma, we can obtain $\sigma_{2} \longrightarrow 0$ as $t \longrightarrow \infty$, and from the definition of $\sigma_{2}$ and $\sigma_{1}$, we can obtain $\sigma_{1} \longrightarrow 0$ and $\mathbf{e}_{1} \longrightarrow 0$, respectively. Therefore, system (15) is globally asymptotic stable with the incremental feedback controller (25) and interactive sliding mode (22).

\section{Adaptive Sliding Mode Attitude Stable Controller Design}

For comparison, an adaptive sliding mode attitude stable controller is designed in this section. The adaptive law is used to estimate unknown moment of inertia online, and the sliding mode controller is used to eliminate external disturbance. Denote $\xi=\left(\Delta J_{11}, \Delta J_{22}, \Delta J_{33}, \Delta J_{12}, \Delta J_{13}, \Delta J_{23}\right)^{\mathbf{T}}$, and $\widehat{\xi}=\left(\Delta \widehat{J}_{11}, \Delta \widehat{J}_{22}, \Delta \widehat{J}_{33}, \Delta \widehat{J}_{12}, \Delta \widehat{J}_{13}, \Delta \widehat{J}_{23}\right)^{\mathbf{T}}$ is the estimation of $\xi$; define error of estimation as $\widetilde{\xi}=\widehat{\xi}-\xi$. Because $-\Delta \mathbf{M}(\theta) \ddot{\theta}-\Delta \mathbf{C}(\theta, \dot{\theta}) \dot{\theta}-\Delta \mathbf{G}(\theta, \dot{\theta})$ is linear with $\xi$, denote 


$$
\begin{aligned}
\mathbf{Y} & =-\Delta \mathbf{M}(\boldsymbol{\theta}) \ddot{\theta}-\Delta \mathbf{C}(\boldsymbol{\theta}, \dot{\theta}) \dot{\theta}-\mathbf{G}_{0}(\boldsymbol{\theta}, \dot{\theta})-\Delta \mathbf{G}(\boldsymbol{\theta}, \dot{\theta}) \\
& =-\mathbf{G}_{0}(\boldsymbol{\theta}, \dot{\theta})+\mathrm{H} \boldsymbol{\xi},
\end{aligned}
$$

$$
\boldsymbol{\sigma}=k_{\mathrm{SL} 1} \mathbf{e}_{1}+\mathbf{e}_{2},
$$

where $H$ is a matrix. Let sliding mode surface as

$$
\begin{aligned}
\dot{\sigma} & =k_{\mathrm{SL} 1} \dot{e}_{1}+\dot{e}_{2} \\
& =k_{\mathrm{SL} 1} \mathbf{e}_{2}+\left[-\mathbf{M}_{0}^{-1} \mathbf{C}_{0} \mathbf{e}_{2}-\mathbf{M}_{0}^{-1} \mathbf{C}_{0} \dot{\theta}_{r}-\ddot{\theta}_{r}+\mathbf{M}_{0}^{-1}\left(\mathbf{u}+\mathbf{d}-\mathbf{G}_{0}(\boldsymbol{\theta}, \dot{\theta})+\mathrm{H} \xi\right)\right] .
\end{aligned}
$$

Design the adaptive sliding mode controller (ASMC) as

$$
\begin{array}{r}
\mathbf{u}=-k_{\mathrm{SL} p} \mathbf{M}_{0} \boldsymbol{\sigma}-\mathrm{H} \hat{\xi}-\mathbf{M}_{0} k_{\mathrm{SL} 1} \mathbf{e}_{2}+\mathbf{C}_{0} \mathbf{e}_{2}+ \\
\mathbf{C}_{0} \dot{\theta}_{r}+\mathbf{M}_{0} \ddot{\theta}_{r}+\mathbf{G}_{0}(\boldsymbol{\theta}, \dot{\theta})-\operatorname{Dsat}(\boldsymbol{\sigma}),
\end{array}
$$

and adaptive law as

$$
\dot{\vec{\xi}}=\Gamma H^{\mathrm{T}} \boldsymbol{\sigma},
$$

where $k_{\mathrm{SL} 1}$ is a constant and $\Gamma$ is a constant matrix.

To verify the global asymptotic stability of closed-loop system, consider Lyapunov function

$$
V=\frac{1}{2} \boldsymbol{\sigma}^{\mathrm{T}} \mathbf{M}_{0} \boldsymbol{\sigma}+\frac{1}{2} \widetilde{\xi}^{\mathrm{T}} \Gamma^{-1} \widetilde{\xi}
$$

Take the time derivative of $V$ and substituting (32) and (33) into derivative, we get

$$
\begin{aligned}
\dot{V}= & \boldsymbol{\sigma}^{\mathrm{T}} \mathbf{M}_{0} \dot{\boldsymbol{\sigma}}+\tilde{\xi}^{\mathrm{T}} \Gamma^{-1} \dot{\hat{\xi}} \\
= & \boldsymbol{\sigma}^{\mathrm{T}} \mathbf{M}_{0}\left\{k_{\mathrm{SL} 1} \mathbf{e}_{2}+\left[-\mathbf{M}_{0}^{-1} \mathbf{C}_{0} \mathbf{e}_{2}-\mathbf{M}_{0}^{-1} \mathbf{C}_{0} \dot{\theta}_{r}-\theta_{r}+\mathbf{M}_{0}^{-1}\left(\mathbf{u}+\mathbf{d}-\mathbf{G}_{0}(\boldsymbol{\theta}, \dot{\theta})+\mathrm{H} \xi\right)\right]\right\}+\widetilde{\xi}^{\mathrm{T}} \Gamma^{-1} \dot{\hat{\xi}} \\
= & \boldsymbol{\sigma}^{\mathrm{T}} \mathbf{M}_{0}\left\{k_{\mathrm{SL} 1} \mathbf{e}_{2}+\left[-\mathbf{M}_{0}^{-1} \mathbf{C}_{0} \mathbf{e}_{2}-\mathbf{M}_{0}^{-1} \mathbf{C}_{0} \dot{\theta}_{r}-\ddot{\theta}_{r}+\mathbf{M}_{0}^{-1}\left(-k_{\mathrm{SL} p} \mathbf{M}_{0} \boldsymbol{\sigma}-\mathrm{H} \hat{\xi}-\mathbf{M}_{0} k_{\mathrm{SL} 1} \mathbf{e}_{2}+\right]\right.\right. \\
& \left.\left.\mathbf{C}_{0} \mathbf{e}_{2}+\mathbf{C}_{0} \dot{\theta}_{r}+\mathbf{M}_{0} \ddot{\theta}_{r}+\mathbf{G}_{0}-D \operatorname{sat}(\boldsymbol{\sigma})+\mathbf{d}-\mathbf{G}_{0}+\mathrm{H} \xi\right]\right\}+\widetilde{\xi}^{\mathrm{T}} \Gamma^{-1} \dot{\bar{\xi}} \\
= & \boldsymbol{\sigma}^{\mathrm{T}} \mathbf{M}_{0}\left\{\left[\mathbf{M}_{0}^{-1}\left(-k_{\mathrm{SL} p} \mathbf{M}_{0} \boldsymbol{\sigma}-\mathrm{H} \hat{\xi}-D \mathbf{s a t}(\boldsymbol{\sigma})+\mathbf{d}+\mathrm{H} \xi\right)\right]\right\}+\widetilde{\xi}^{\mathrm{T}} \Gamma^{-1} \dot{\vec{\xi}} \\
= & -k_{\mathrm{SL} p} \boldsymbol{\sigma}^{\mathrm{T}} \mathbf{M}_{0} \boldsymbol{\sigma}-\boldsymbol{\sigma}^{\mathrm{T}}(D \mathbf{s a t}(\boldsymbol{\sigma})-\mathbf{d})+\widetilde{\xi}^{\mathrm{T}}\left(\Gamma^{-1} \dot{\vec{\xi}}-\mathrm{H}^{\mathrm{T}} \boldsymbol{\sigma}\right) \\
= & -k_{\mathrm{SL} p} s^{\mathrm{T}} \mathbf{M}_{0} \boldsymbol{\sigma}-\boldsymbol{\sigma}^{\mathrm{T}}(D \mathbf{s a t}(\boldsymbol{\sigma})-\mathbf{d}) \leq-k_{\mathrm{SL} p} \boldsymbol{\sigma}^{\mathrm{T}} \mathbf{M}_{0} \boldsymbol{\sigma} .
\end{aligned}
$$

Then, $\quad \dot{V} \leq-k_{\mathrm{SL} p} \sigma^{\mathrm{T}} \mathbf{M}_{0} \sigma \leq 0$; therefore, the adaptive sliding mode controller (32) and (33) ensures the global asymptotic stability of the closed-loop system (15).

\section{Numerical Simulation}

5.1. Performance Comparison between the above Two Controllers. In this section, the iterative sliding mode and increment feedback controller (ISMIFC) is compared with the adaptive sliding mode controller (ASMC) in the environment of spacecraft on-orbit capturing noncooperation target. The initial attitude angle is $\theta_{0}=\left(10^{\circ}, 10^{\circ}, 10^{\circ}\right)^{\mathrm{T}}$, and correspondingly, angular velocity is $\dot{\theta}_{0}=(0,0,0)^{\mathrm{T}}$, orbital rate is $\omega_{0}=0.01 \mathrm{rad} / \mathrm{s}$, and nominal inertial matrix is $\mathbf{J}_{0}=\left[\begin{array}{ccc}2000 & 120 & 90 \\ 120 & 1700 & 140 \\ 90 & 140 & 1500\end{array}\right]$.
The desired attitude angle is $\theta_{r}=\left(1^{\circ}, 1^{\circ}, 1^{\circ}\right)^{\mathrm{T}}$, desired angle velocity is $\dot{\theta}_{r}(0)=(0,0,0)^{\mathrm{T}}$, and acceleration is $\ddot{\theta}_{r}(0)=(0,0,0)^{\mathrm{T}}$. The external disturbance is $\mathbf{d}=(\sin (t), \sin (t), \sin (t))^{\mathrm{T}}$, and the perturbation matrix of moment of inertia is $\Delta \mathbf{J}=0.001 \times \mathbf{J}_{0} \times t$.

When the control parameters are set as $k_{1}=1.2, k_{2}=1$, $k_{3}=0.008, k_{4}=1, k_{p}=1.5, \varepsilon=0.1$, and the time of simulation is set as $800 \mathrm{~s}$; the control based on incremental feedback controller is simulated. Under the iterative sliding mode and increment feedback controller, the attitude angle tracking error histories and control input torque are shown in Figures 1 and 2, respectively.

The adaptive sliding mode controller is simulated, control parameters are set as $k_{\mathrm{SL} p}=1.5, k_{\mathrm{SL} 1}=1.2, D=1$, $\Gamma=\operatorname{diag}(3,3,3,3,3,3)$, and $\widehat{\xi}_{0}=[0,0,0,0,0,0]^{\mathrm{T}}$, and the time of simulation is set as $10 \mathrm{~s}$. The attitude angle tracking 


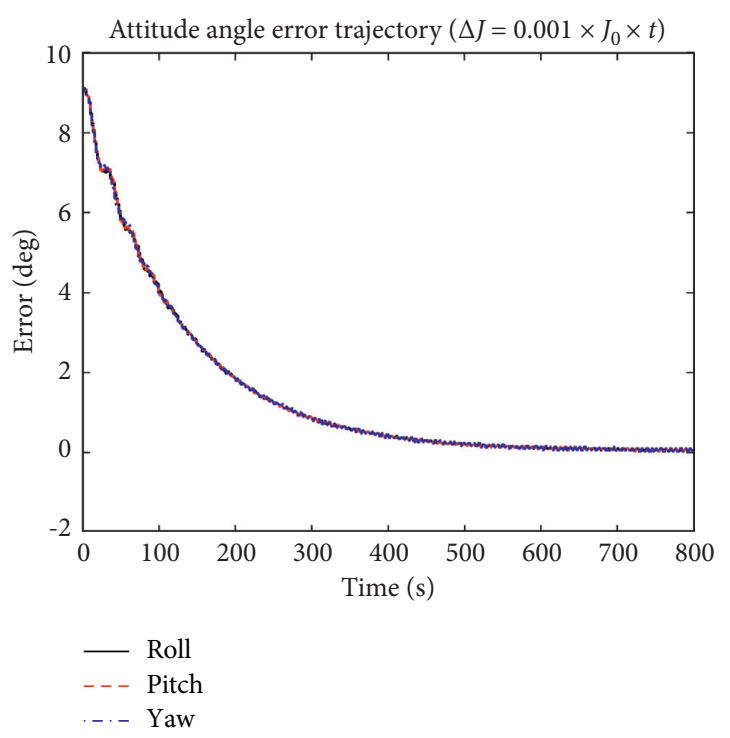

FIgUre 1: Attitude angle tracking error with ISMIFC $\left(\Delta \mathbf{J}=0.001 \times \mathbf{J}_{0} \times t\right)$.
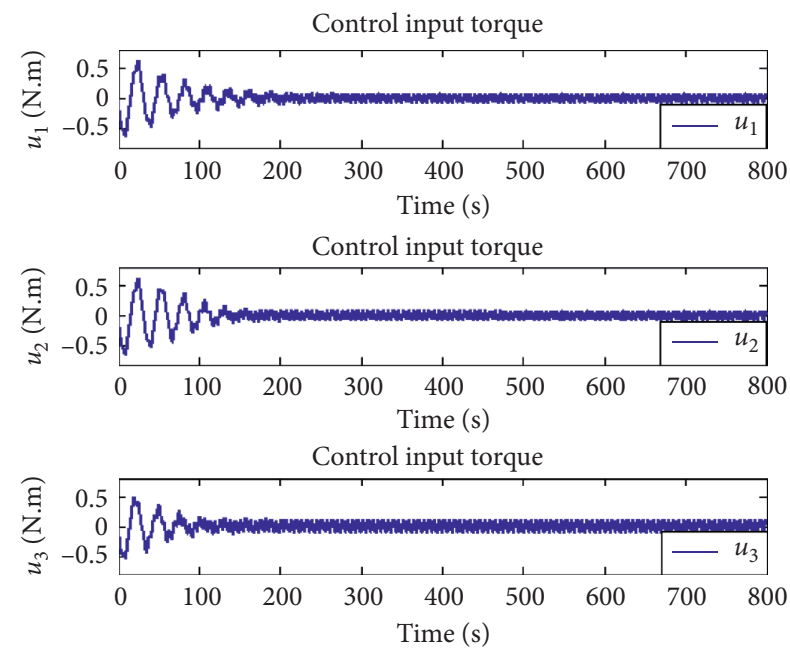

FIgURE 2: Control input torque with ISMIFC $\left(\Delta \mathbf{J}=0.001 \times \mathbf{J}_{0} \times t\right)$.

error trajectory and control input torque of the adaptive sliding mode controller are shown in Figures 3 and 4, respectively.

Comparing with Figures 1 and 3, it can see that the attitude angle of the two controllers decreases rapidly, but the convergence time of adaptive sliding mode controller is shorter than that of the iterative sliding and increment feedback controller, the convergence time of adaptive sliding mode controller is being about $7 \mathrm{~s}$, and the iterative sliding controller is being about $700 \mathrm{~s}$. In the process of attitude stability control, the control time is not a strict restrain, and the convergence time of 700 second is not long. Figures 2 and 4 show the control torque of two controllers, respectively. In Figure 2, the control torque is between $-0.6 \mathrm{~N} \cdot \mathrm{m}$ and $0.6 \mathrm{~N} \cdot \mathrm{m}$ at the early stage and stabilizes at zero. In Figure 4 , the control torque ranges from $-550 \mathrm{~N} \cdot \mathrm{m}$ to $200 \mathrm{~N} \cdot \mathrm{m}$ and gradually declines to zero. Compared with the

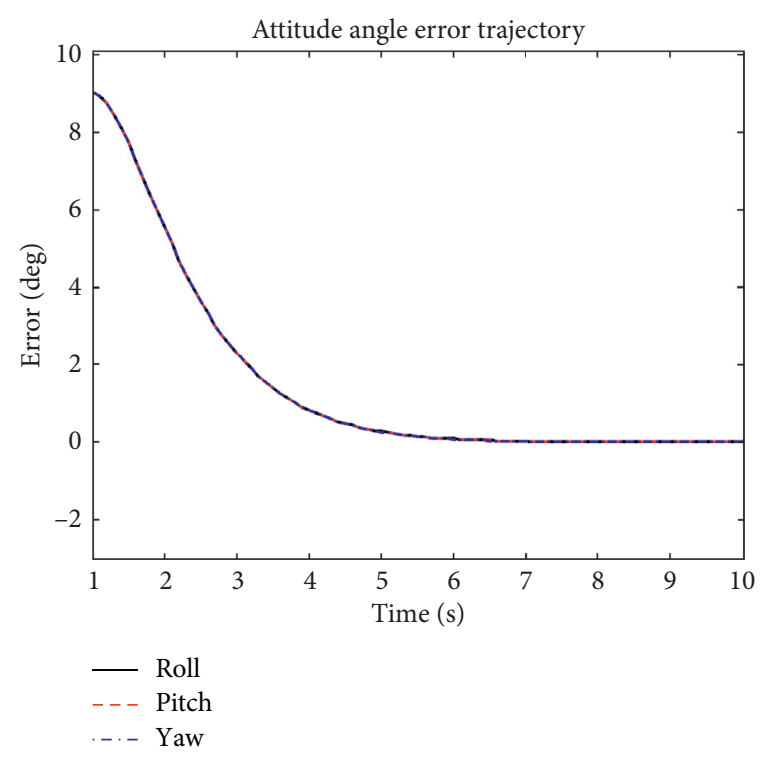

Figure 3: Attitude angle tracking error with ASMC $\left(\Delta \mathbf{J}=0.001 \times \mathbf{J}_{0} \times t\right)$.
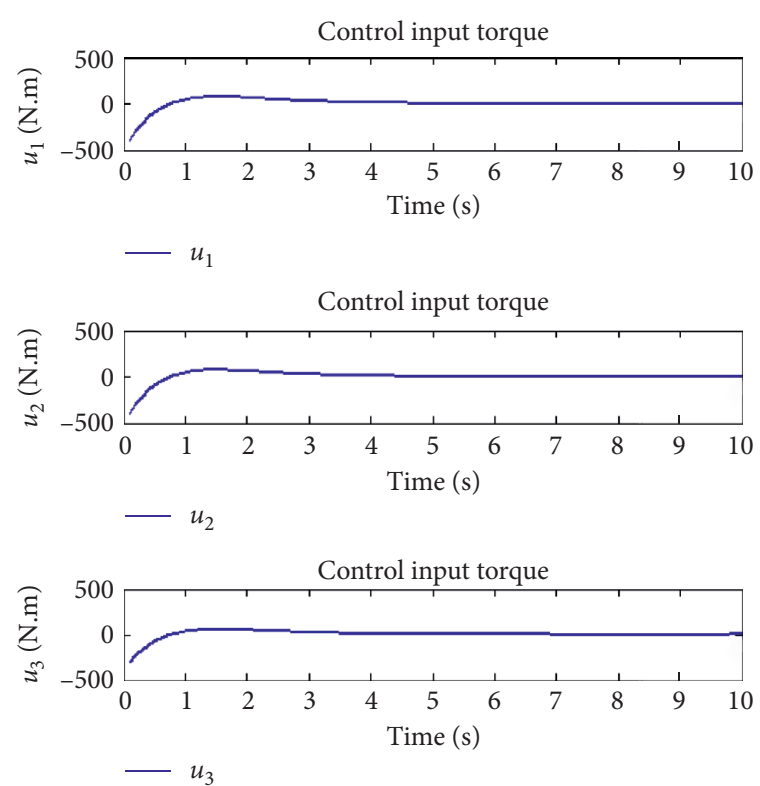

Figure 4: Control input torque with $\operatorname{ASMC}\left(\Delta \mathbf{J}=0.001 \times \mathbf{J}_{0} \times t\right)$.

control torques of two controllers, the control input of iterative sliding controller consumes less than adaptive sliding controller, so the iterative sliding controller is more suitable in practical spacecraft attitude controller.

5.2. Robust Analysis of Iterative Sliding and Increment Feedback Controller. From Figures 5 to 8, the trajectory of attitude, angle tracking error, and control input torque are given.

In Figures 5 and 6, the attitude angle error and control input torque are given by the perturbation matrix $\Delta \mathbf{J}=0.01 \times \mathbf{J}_{0} \times t$, respectively. When the perturbation 


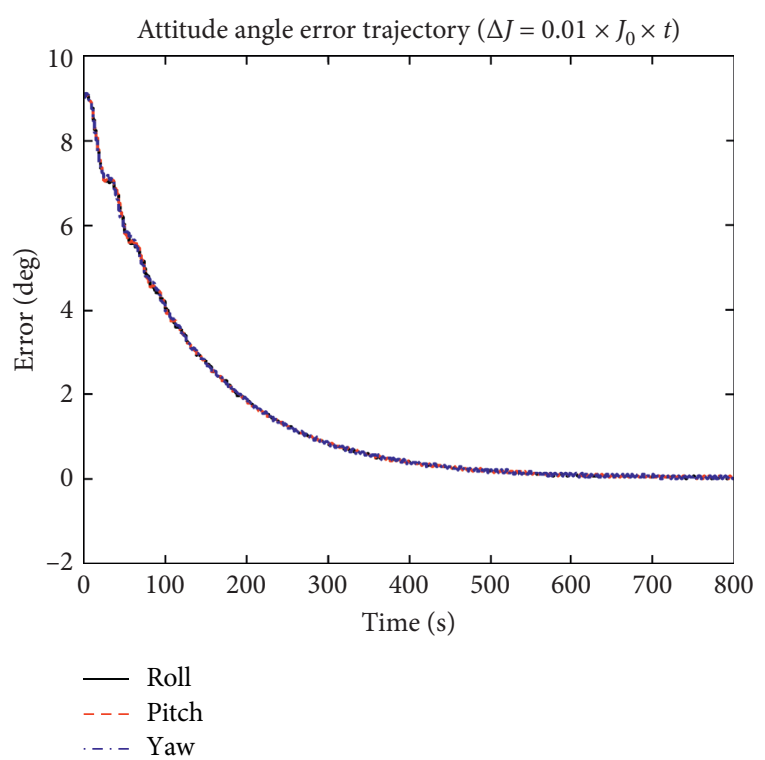

FIgURE 5: Attitude angle tracking error with ISMIFC $\left(\Delta \mathbf{J}=0.01 \times \mathbf{J}_{0} \times t\right)$.

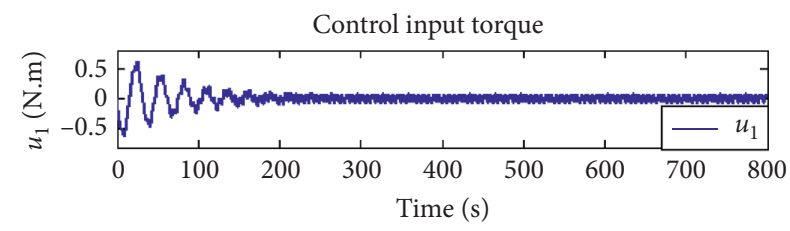

Control input torque

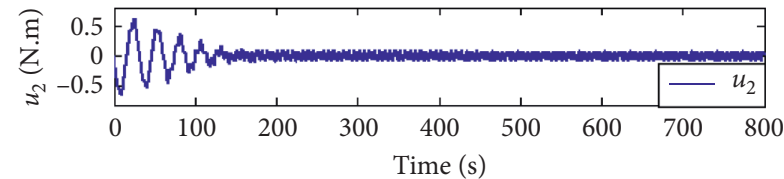

Control input torque

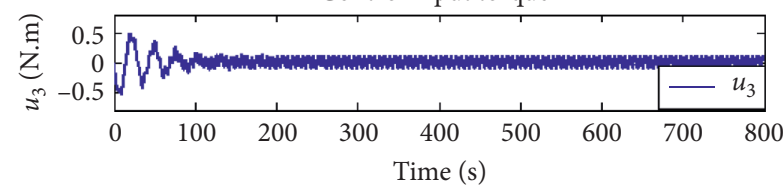

FIGURE 6: Control input torque with ISMIFC $\left(\Delta \mathbf{J}=0.01 \times \mathbf{J}_{0} \times t\right)$.

matrix is $\Delta \mathbf{J}=0.1 \times \mathbf{J}_{0} \times t$, the attitude angle error and control input torque are shown in Figures 7 and 8, respectively.

Compared with Figures 1, 5, and 7, it can be seen that the convergence time and the convergence curve are not the same. It can be seen from Figures 2, 6, and 8, the control input torque curve is similar.

In conclusion, the convergence speed, convergence time, and control input torque of system are not affected by inertia disturbance. It is shown that the incremental feedback controller is robust to parameter perturbation and is suitable for spacecraft attitude stability control in the process of onorbit capturing noncooperative target.

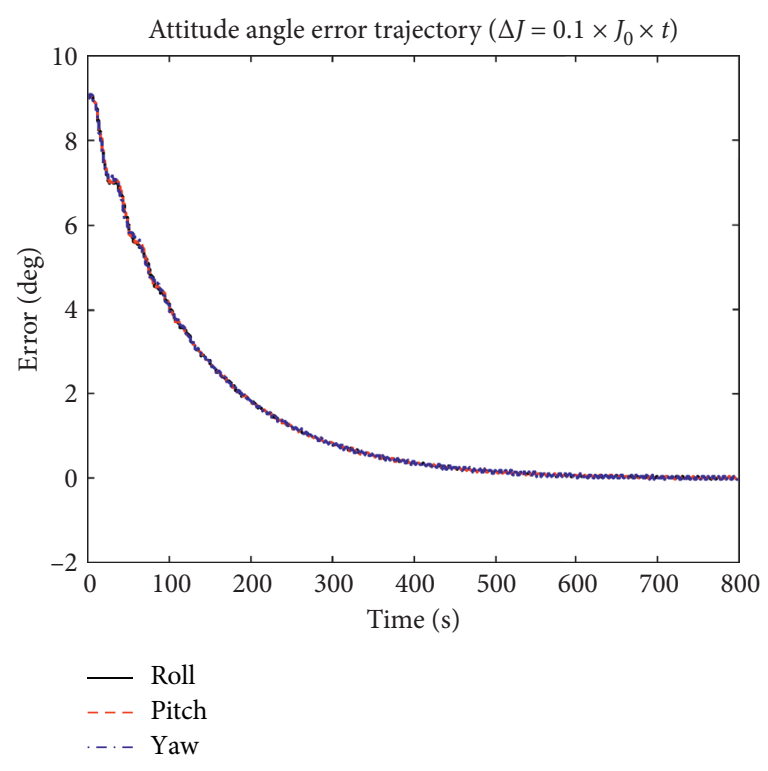

Figure 7: Attitude angle tracking error with ISMIFC $\left(\Delta \mathbf{J}=0.1 \times \mathbf{J}_{0} \times t\right)$.

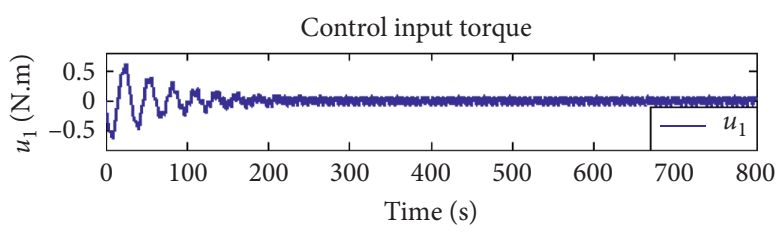

Control input torque

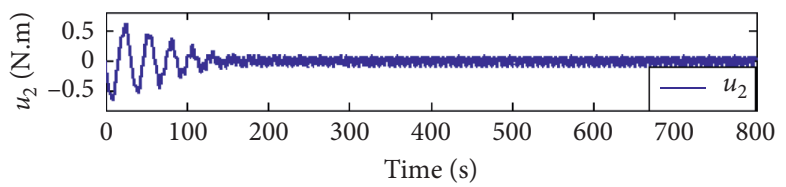

Control input torque

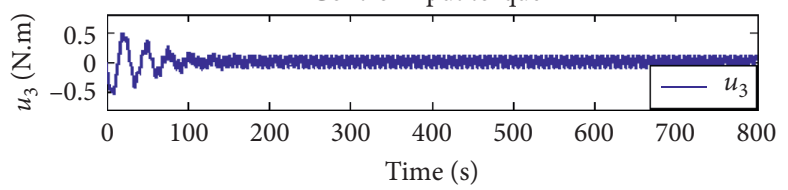

FIgURE 8: Control input torque with ISMIFC $\left(\Delta \mathbf{J}=0.1 \times \mathbf{J}_{0} \times t\right)$.

\section{Conclusion}

The attitude stability control problem of spacecraft onorbit capturing noncooperative target is analyzed, and a globally asymptotic stable nonlinear controller based on nonlinear iterative sliding mode and increment feedback control is proposed. The controller does not need to estimate the moment of inertia and external disturbances, and the control input torque is small due to the use of utilization of the bounded sliding mode surface. The simulation results show that the controller is robust for parameter variations with fast convergence speed and less control input torque. 


\section{Data Availability}

The data used to support the findings of this study are included within the article.

\section{Conflicts of Interest}

The authors declare that there are no conflicts of interest in connection with the work submitted.

\section{Authors' Contributions}

CW participated in the controller design and theoretical proof and drafted the manuscript. ZQ and HX participated in the numerical simulation of the study and performed the statistical analysis. LJ and YJ conceived of the study and participated in its design and coordination and helped to draft the manuscript. All authors read and approved the final manuscript.

\section{Acknowledgments}

This work was supported by the Natural Science Foundation Of Shaanxi under project 2020JM-488, Shanghai Rising Star Program under 20QA1404300, Youth Fund Project of Xi' an University of Architecture and Technology under QN1727, Talent Fund Project of Xi'an University of Architecture and Technology under 6040317017, Innovation and Entrepreneurship Training Program Project of Shaanxi under S202010703154, and Education Department Special Scientific Research Project of Shaanxi under 20JK0728.

\section{References}

[1] E. Silani and M. Lovera, "Magnetic spacecraft attitude control: a survey and some new results," Control Engineering Practice, vol. 13, no. 3, pp. 357-371, 2005.

[2] Y. Yang, "Spacecraft attitude determination and control: quaternion based method," Annual Reviews in Control, vol. 36, no. 2, pp. 198-219, 2012.

[3] Y. Yang, "Quaternion-based LQR spacecraft control design is a robust pole assignment design," Journal of Aerospace Engineering, vol. 27, no. 1, pp. 168-176, 2014.

[4] C. D. Yang and Y. Sun, "Mixed $\mathrm{H}_{2} / \mathrm{H}_{\infty}$ state-feedback design for microsatellite attitude control," Control Engineering Practice, vol. 10, pp. 951-970, 2002.

[5] C.-H. Won, "Comparative study of various control methods for attitude control of a LEO satellite," Aerospace Science and Technology, vol. 3, no. 5, pp. 323-333, 1999.

[6] C. Li, K. L. Teo, B. Li, and G. Ma, "A constrained optimal PIDlike controller design for spacecraft attitude stabilization," Acta Astronautica, vol. 74, no. 74, pp. 131-140, 2012.

[7] Y. Wang, K. Li, K. Yang, and H. Ji, "Adaptive backstepping control for spacecraft rendezvous on elliptical orbits based on transformed variables model," International Journal of Control, Automation and Systems, vol. 16, no. 1, pp. 189-196, 2018.

[8] P. Chutiphon, "Extended state observer-based third-order sliding mode finite-time attitude tracking controller for rigid spacecraft sciece China," Information Sciences, vol. 62, no. 1, pp. 9389-9399, 2019.
[9] B. Huang, A.-J. Li, Y. Guo, and C.-Q. Wang, "Fixed-time attitude tracking control for spacecraft without unwinding," Acta Astronautica, vol. 151, pp. 818-827, 2018.

[10] F.-K. Yeh, "Sliding-mode adaptive attitude controller design for spacecrafts with thrusters," IET Control Theory \& Applications, vol. 4, no. 7, pp. 1254-1264, 2010.

[11] K. Lu and Y. Xia, "Adaptive attitude tracking control for rigid spacecraft with finite-time convergence," Automatica, vol. 49, no. 12, pp. 3591-3599, 2013.

[12] L. Sun and Z. Zheng, "Adaptive relative pose control of spacecraft with model couplings and uncertainties," Acta Astronautica, vol. 143, no. 143, pp. 29-36, 2018.

[13] Y. Park, "Inverse optimal and robust nonlinear attitude control of rigid spacecraft," Aerospace Science and Technology, vol. 28, no. 1, pp. 257-265, 2013.

[14] Z. Zhuo, Z. Zhang, and Z. Hui, "Distributed attitude control for multispacecraft via takagi-sugeno fuzzy approach," IEEE Transactions on Aerospace and Electronic Systems, vol. 54, no. 2, pp. 642-654, 2018.

[15] X. Cao, P. Shi, Z. Li, and M. Liu, "Neural-network-based adaptive backstepping control with application to spacecraft attitude regulation," IEEE Transactions on Neural Networks and Learning Systems, vol. 29, no. 9, pp. 4303-4313, 2018.

[16] A. A. Chunodkar and M. R. Akella, "Switching angular velocity observer for rigid-body attitude stabilization and tracking control," Journal of Guidance, Control, and Dynamics, vol. 37, no. 3, pp. 869-878, 2014.

[17] G. Hu, B. Gao, Y. Zhong, and C. Gu, "Unscented kalman filter with process noise covariance estimation for vehicular ins/gps integration system," Information Fusion, vol. 64, pp. 194-204, 2020.

[18] G. Hu, "A new direct filtering approach to INS/GNSS integration," Aerospace Science and Technology, vol. 77, pp. 755-764, 2018.

[19] L. Song, Z. Duan, B. He, and Z. Li, "Application of federal kalman filter with neural networks in the velocity and attitude matching of transfer alignment," Complexity, vol. 2018, no. 2, pp. 1-7, 2018.

[20] D. A. Munoz and W. Marquardt, "Robust control dedign of a class of nonlinear input- and state- constrained systems," Annual Review in Control, vol. 37, pp. 232-245, 2013.

[21] J. Tao, T. Zhang, and Y. Nie, "Adaptive prescribed performance control for flexible spacecraft with input saturation and actuator misalignment," Tsinghua Science and Technology, vol. 24, no. 6, pp. 694-705, 2019. 